\title{
Research on submarine cable detection technology based on information fusion
}

\author{
Zhou Xue-jun $^{1 \mathrm{a}}$, Zhang Yang ${ }^{1 \mathrm{~b}}$, Zuo Ming-jun $^{1}$, Yuan Zhen-dong $^{2}$ \\ ${ }^{1}$ Electronic Engineering College, Naval University of Engineering, Wuhan 430033, China \\ ${ }^{2}$ The Fourth of the China Sea Fleet Command, Zhanjiang 524001,China \\ a Xuejun-Zhou@163.com, ${ }^{\text {b }}$ zhangyang.990@163.com
}

Keywords: submarine cable; information fusion; detection

\begin{abstract}
Based on information fusion, analyzed submarine cable probe positioning technology and methods, through the information classification and information correlation in submarine cable detection process, proposed submarine cable five parallel-type information fusion method, improved information processing capacity and technical updates of submarine cable detection.
\end{abstract}

\section{Introduction}

With the increase of the international information exchange, the submarine cable growing at an unprecedented rate of rapid construction, however with the increase in the amount of laying submarine cable, submarine cable maintenance support for the detection and location technology has been a technology focus and difficulty of coastal states in research ${ }^{[1]}$. China is relatively backward in research submarine cable fault detection and location technology, this difference is not only due to the low detection equipment accuracy, but the main reason is the detecting process and the routing decision is often by experience, large artificial subjective factors, the lack of information integration, information collection, information processing, information processing and other auxiliary judge technology in the detection. Aiming submarine cable detection process classified information flow analysis and parsed information integration, promote update and improve submarine cable detection technology.

\section{Submarine cable detection method}

Submarine cable detection positioning technologies including sonar detection method, active magnetic detection method, passive magnetic detection method ${ }^{[2]}$. Sonar detection method is based on the reflected sonar signal of submarine cable, including sonar shallow profile and sides can sonar. However, due to the limitations of complex geological environment and seabed sonar technology, sonar detection method is not very satisfactory. Active magnetic detection method is via submarine cable conductor to a specific frequency of the input AC signal, causing the surrounding electromagnetic signals to strengthen the broader application based on electromagnetic induction method, but this method attenuation is big, the signal is weak, is not suitable for long-distance submarine cable detection ${ }^{[3]}$. The passive magnetic detection technology is based on methods of detecting the physical magnetic properties of submarine cable, which does not require strengthen the signal of submarine cable, complex deep water submarine cable detection, this method is suitable for the detection of complex waters, enriching the submarine cable detection method, improving the submarine cable detection technology and performance. Several detection methods have advantages and disadvantages, submarine cable project often use a variety of detection equipment and technology co-detection.

\section{Submarine cable detection system based on information fusion}

\subsection{Fusion analysis}

Detection system based on structural information fusion technology to process data, obtains multi-level information, and enhances the ability of information processing and integration of 
systems can make better decision-making basis for the operator. In the submarine cable detection systems, the information is mainly transmitted and collected by various sensors, to improve the overall performance of submarine cable detection system, we can deal with this information in the data layer, feature layer and decision-making by information fusion technology ${ }^{[5]}$.

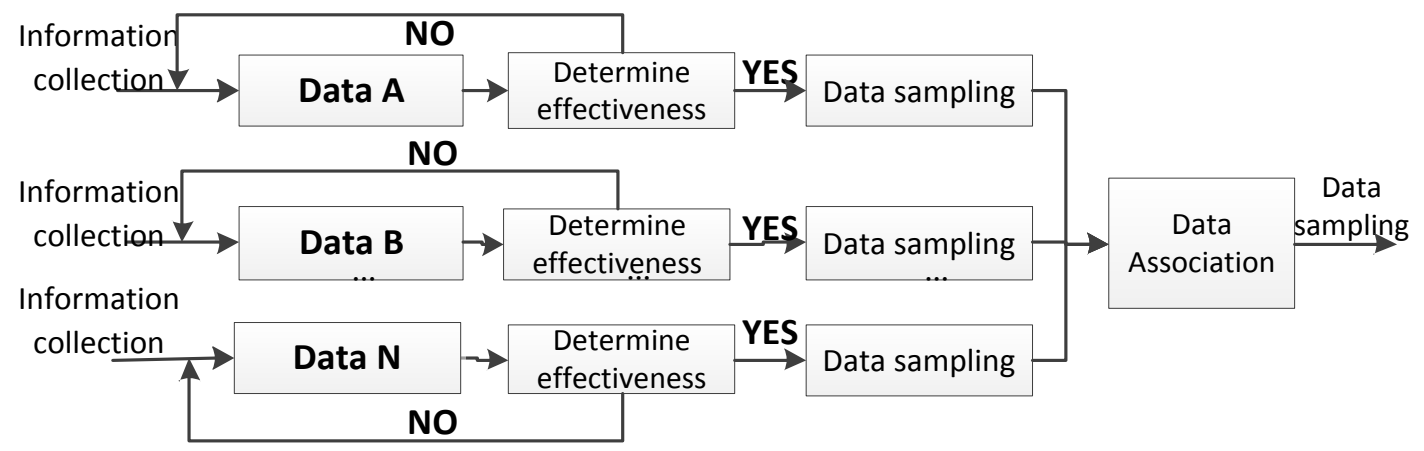

Fig.3 Data layer information fusion diagram

For the system architecture of submarine cable detection, data layer can design parallel systems integration on the target sea, five target submarine, navigation system, power systems and detection equipment. Data integration layer is mainly for simple data analysis and comprehensive treatment of the raw data according to various information layers of submarine cable detection system sensors. This preliminary data integration can improve the relevance of the data, remove the error collection information, improve the efficiency of information processing system, the principle shown in Figure 3.

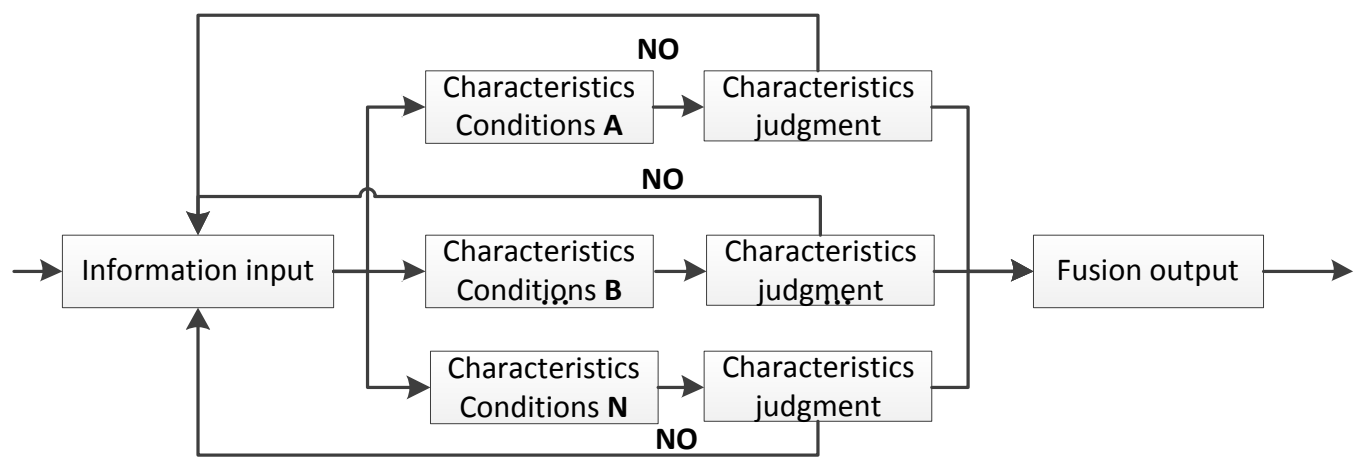

Fig.4 Feature level information fusion diagram

Submarine cable feature level fusion is mainly on feature extraction of information, beneficial the submarine cable for target recognition module integrated information, system according features database remove errors and useless information of detection systems, improve the practical value of information, the principle shown in Figure 4.

Decision Fusion using the decision library for the decision make and judgment. The main types of judgment contain submarine cable routing judgment and undesired point identification, the system can display graphically based on the identification result of determination, provide the optimal decision for the operator, the principle shown in Figure 5.

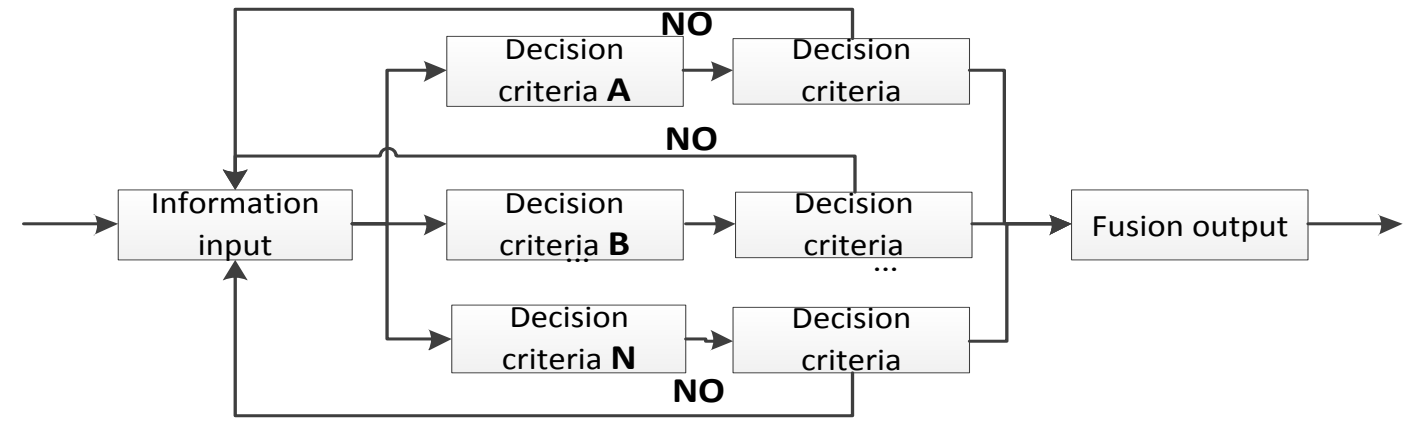

Fig.5 Decision-making information fusion diagram 


\subsection{Fusion Design}

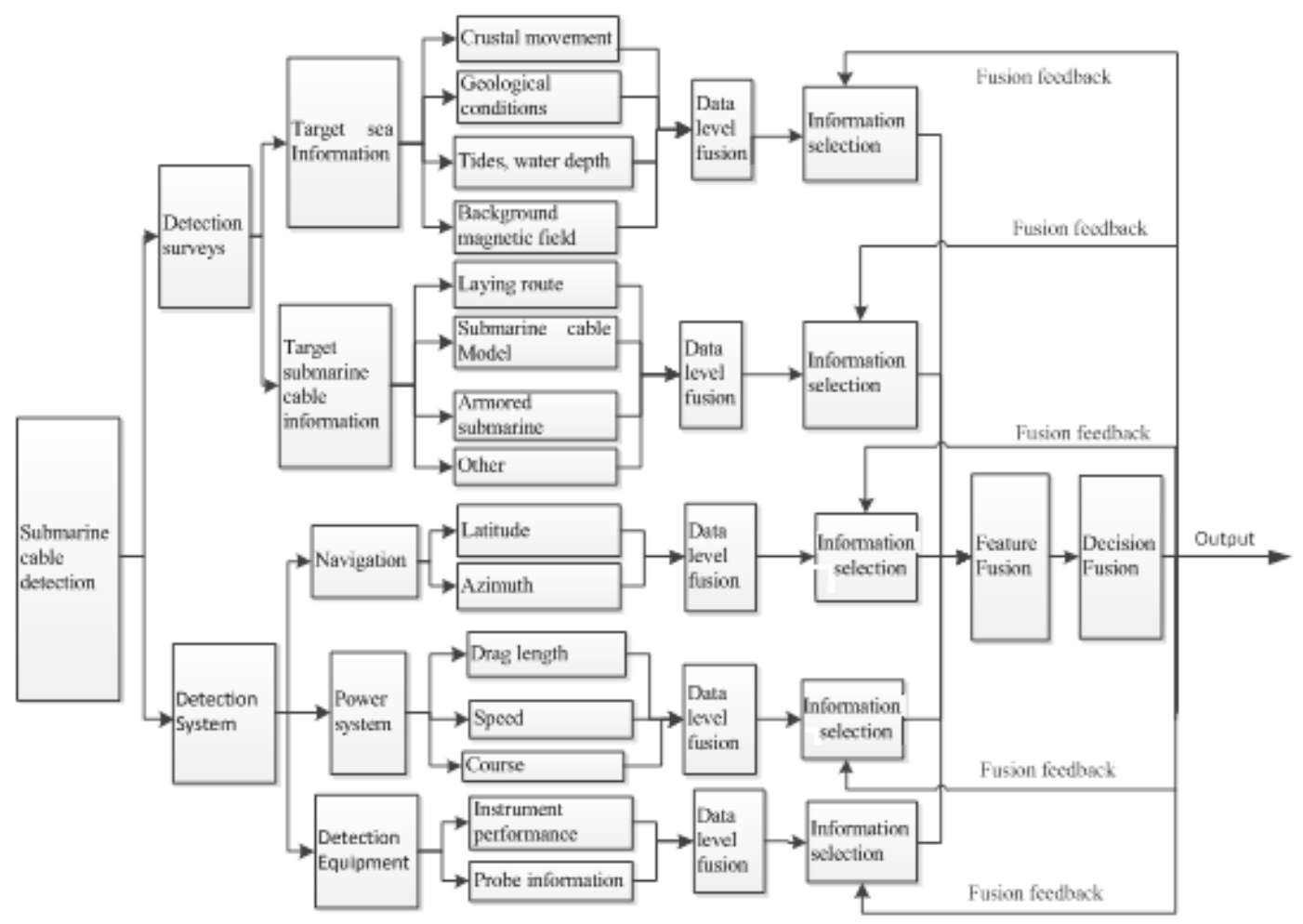

Fig.6 Submarine cable detection system information fusion design drawing

submarine cable detection process can divide into detection surveys and detection implemented two parts. Detection surveys are preparatory work of submarine cable detection, mainly through various means and methods, survey crustal movement, geological conditions, tides, water depth, background geomagnetic field of sea area. And laying route, submarine cable model, submarine cable armored condition, submarine cable fault type of submarine cable, forming a preliminary survey database. Detection implemented involves all kinds of information and data, including latitude and longitude of navigation, speed, heading, sonar detectors, electromagnetic detection and other information.

Information fusion feedback is conducive to detect personnel decision-making based on information fusion result of the detection system control, select information data layer fused to processing and optimization, enhance the interaction and coordination of information, optimize the system structure, enhance operability of the system.

According to the structure characteristics of submarine cable detection system and detection of comprehensive factors, according to the theory of information fusion system is designed with multitier distributed submarine cable feedback function detection fusion structure, submarine cable detection system information fusion process as shown in Figure 6.

\section{Summary}

The information analysis and processing in the submarine cable detection, with design based on information fusion, improved the efficiency of information processing, enhanced system intelligent level, promoted the submarine cable detection technology progress and development.

\section{References}

[1] Yang Z,Xuejun Z,Zhen Z.Passive Magnetic Detection of Optical Fiber Submarine Cable[C].Review Form of the 2014 3rd International Symposium on Computer, Communication, Control and Automation,2014:2697-2701.

[2] Mingjiu Z,Feng T,Xiaorui Q.Research of parallel placed submarine cable route detection 
method[C].3rd International Conference on Image Analysis and Signal Processing,IASP 2011,2011:595-599.

[3] Xuejun Z,Yingjian W,Yuanyuan Z.Research on modeling and experimentation on detecting buried depth of optical fiber submarine cables based on active detection technique[C].Proceedings of the 2009 9th International Conference on Electronic Measurement \& Instruments,2009:91-94.

[4] RenLaiping,Fan Long,Lin Yong,et al.Underwater Depth Controlling Method of Marine Magnetometer Towfish[J].Hydrographic Surveying and Charting,2013,33(2):6-12.

[5] Ding Kai.Research of information fusion technology in ship integrated navigation system[D].Harbin Engineering University. 